Trauma Berufskrankh 2010 · 12[Suppl 3]:323-328 DOI 10.1007/s10039-009-1586-y

Online publiziert: 27. Februar 2010

(c) Springer-Verlag 2010
F.F. Fernandez $\cdot 0$. Eberhardt

Orthopädische Klinik, Olgahospital, Klinikum Stuttgart

\section{Klassifikationen von Frakturen im Kindesalter}

ausgereiften Skeletts verwendet, wodurch die Kenntnisse über die „natural history“ der unterschiedlichen Frakturen erweitert werden konnten. Dies hatte zur Folge, dass die Ursachen für Komplikationen analysiert und systematisch ausgewertet und damit deren Häufigkeit reduziert werden konnten, was auch in einer Senkung der Behandlungskosten resultierte. Therapeutische Maßnahmen wurden effektiver und effizienter. Dennoch kann die AO-Klassifikation des Erwachsenen aus verschiedenen Gründen nicht unverändert für die Frakturen des wachsenden Skeletts übernommen werden.

\section{Kindspezifische Faktoren}

Im Kindesalter ist die Frakturgeometrie im Wesentlichen vom Unfallmechanismus unabhängig. Das Verletzungsmuster wird entscheidend vom Alter und Reifestadium der Wachstumsfugen geprägt, so treten beim Kind kaum Trümmerfrakturen von langen Röhrenknochen oder komplexe Gelenkfrakturen auf.

Im Gegensatz zum Erwachsenen lassen sich bei Frakturen im Kindesalter anhand der Verletzung keine hierarchische Struktur der Brüche und somit auch keine Therapiemethode ableiten. Die Thera- pieform wird im Wesentlichen bestimmt durch:

- das Alter des verunfallten Kindes,

- die Frakturlokalisation,

- die Dislokation,

- die Geometrie sowie

- Begleitverletzungen.

So wird beispielsweise eine Oberschenkelschaftfraktur bei einem 1-jährigen Jungen meist konservativ versorgt, während bereits beim 4-Jährigen eine Operation notwendig sein wird.

Nach Verletzungen des wachsenden Skeletts des Kindes kann es zu Wachstumsstörungen kommen. Inwieweit diese tatsächlich auftreten, hängt von verschiedenen Faktoren ab:

\section{Alter des Kindes zum Unfallzeitpunkt.}

Lokalisation der Fraktur. Neben der eigentlichen Lokalisation sind insbesondere die Nähe der Fraktur zur bzw. eine evtl. Mitbeteiligung der Wachstumsfuge von Bedeutung. So gehen Fugenverletzungen an den unteren Extremitäten gegenüber Verletzungen an den oberen $\mathrm{Ex}$ tremitäten häufiger mit Wachstumsstörungen einher.
Für Frakturen im Kindesalter existieren viele Einteilungen, jedoch alle mit dem entscheidenden Nachteil, dass sie nur für die jeweilige Region entwickelt wurden. Darüber hinaus finden sich für einige Frakturlokalisationen mehrere Klassifikationen z. B. für Ellenbogenfrakturen.

Aufgrund des Fehlens einer systematischen kindspezifischen Klassifikation wurde häufig die AO-Klassifikation des

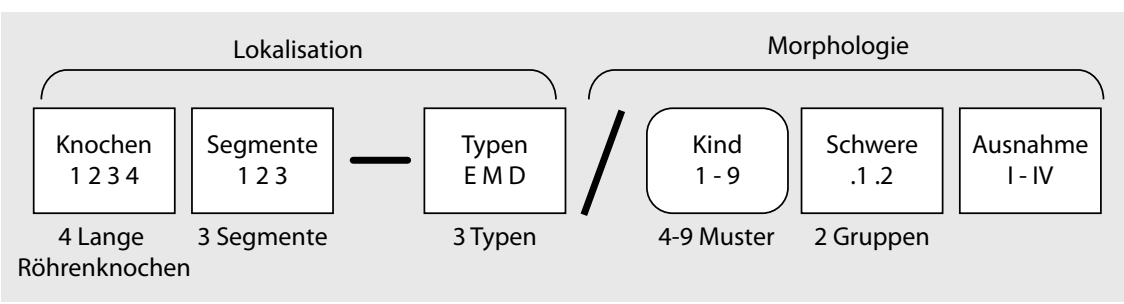

Abb. $1 \Delta$ Prinzip der Frakturklassifikation im Kindesalter. (Aus [4]) 


\title{
Frakturen bei Kindern und Jugendlichen
}

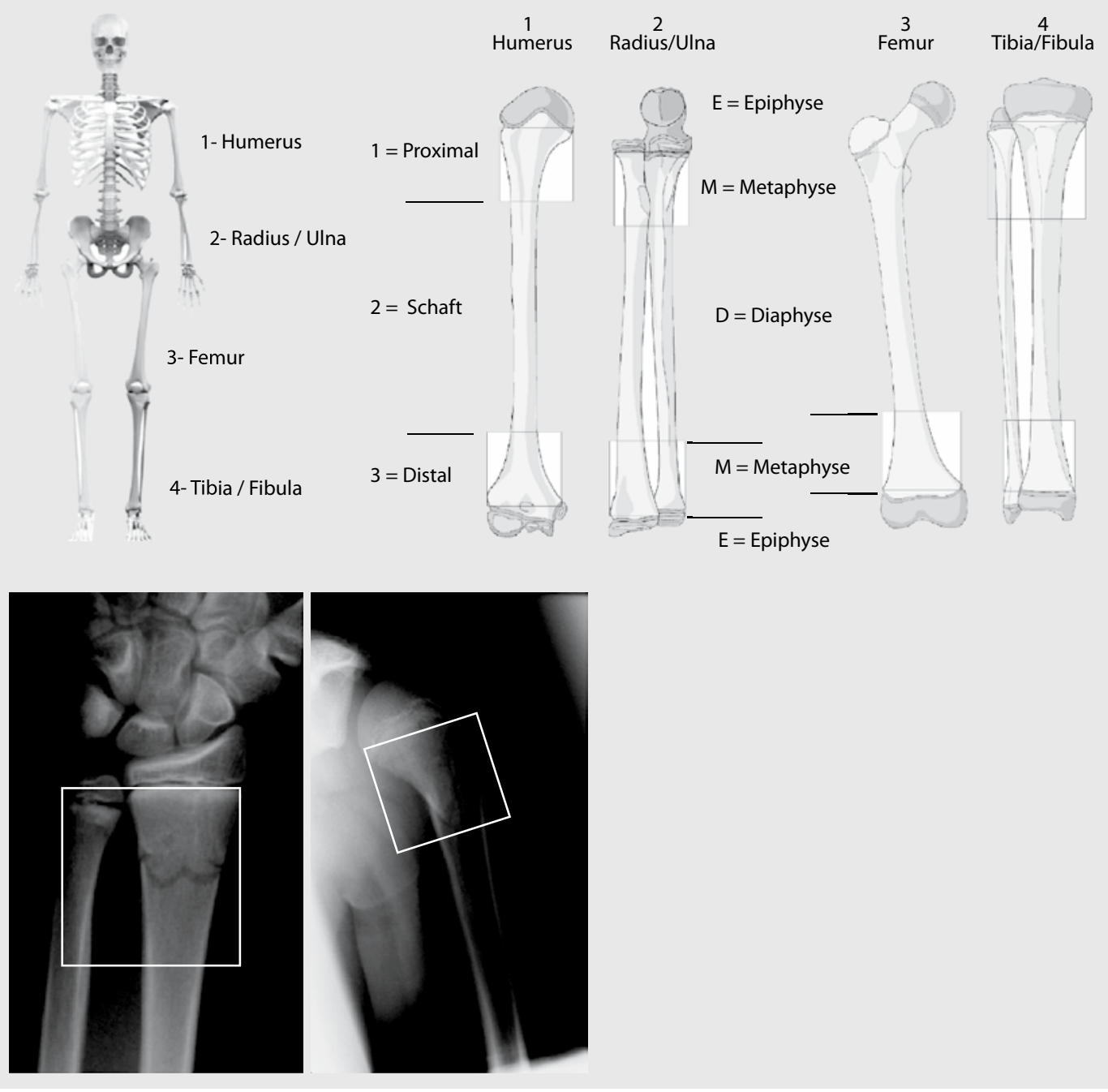

\begin{abstract}
Abb. $2 \varangle$ Bezeichnung der langen Röhrenknochen mit 1-4 in Anlehnung an die Erwachsenen-Müller-AOKlassifikation; Segmentkode: 1-3 für proximal/diaphysär (Schaft)/distal; Typkode: Buchstaben $E, D$ und $M$ für Epi-, Dia- und Metaphyse; weitere Erläuterungen s. Text. (Aus [4])
\end{abstract}

\section{M /2 Inkomplette Fraktur}

(Wulst-, Spiral- oder Grünholzfraktur)
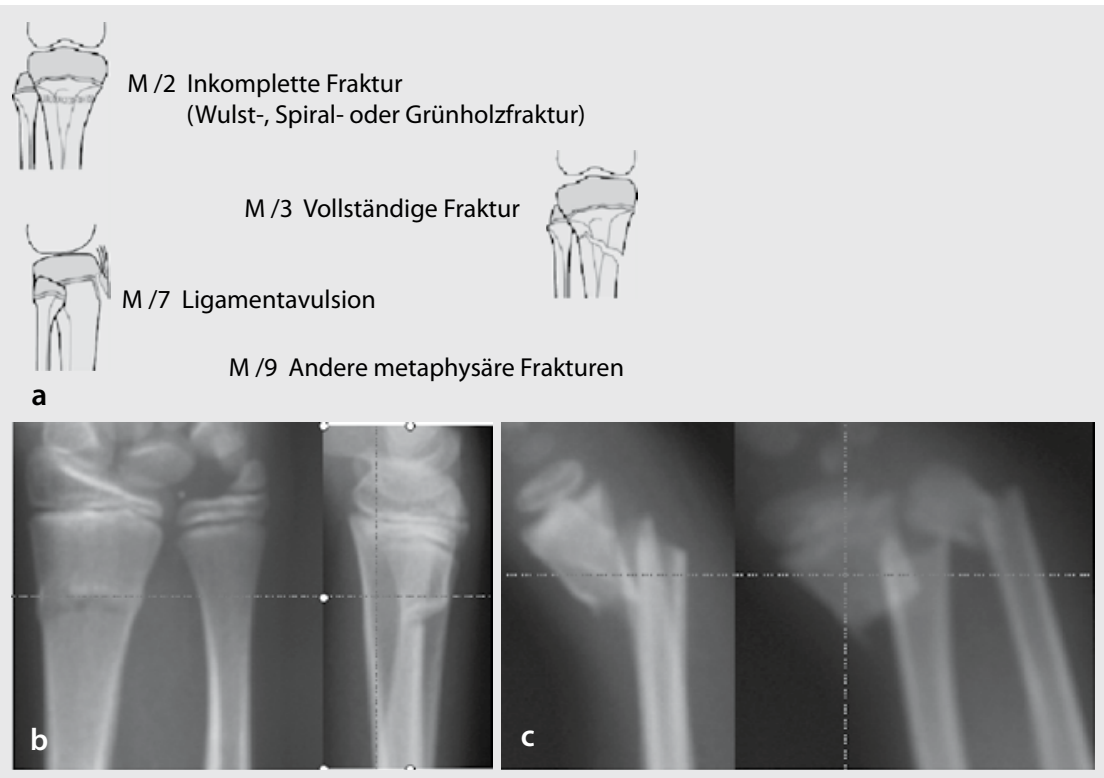

Abb. $3 \Delta$ Metaphysäre Fraktur, a Einteilung, b metaphysäre Fraktur Typ M/2 (inkomplette Fraktur), c vollständige metaphysäre Fraktur Typ M/3. (Aus [4])
Noch vorhandenes Wachstum. Ein solches kann zu einer "Spontankorrektur“ von verbliebenen Fehlstellungen oder durch hemmende Einflüsse zum einseitigen bzw. vorzeitigen Wachstumsfugenverschluss führen. Wachstumsstörungen im Sinne einer Steigerung des Wachstums führen ebenfalls zu Fehlstellungen wie beim Cubitus varus nach Condylusradialis-Frakturen.

\section{Wachstumsbeteiligung der jeweiligen} Epiphysenfuge. Je reifer die Epiphysenfugen sind, desto geringer ist die Gefahr einer Wachstumsstörung.

Die Wachstumsprognose hängt somit nicht allein von der Frakturgeometrie innerhalb der Epiphysenfuge ab. Es scheint keine hierarchische Ordnung der Wachstumsprognosen und eventueller Folgen von Wachstumsstörungen zu geben.

Zweck und Ziel einer kinderspezifischen Klassifikation soll es sein, über 
retrospektive und prospektive Daten, am sinnvollsten jedoch über prospektive Multicenterstudien Daten zusammenzuführen, um evidenzbasierte Therapieempfehlungen abgeben zu können.

In den letzten Jahren wurde an 2 systematischen kinderspezifischen Klassifikationen gearbeitet, die inzwischen validiert wurden. Es handelt sich um

- die Klassifikation der AO Paediatric Expert Group (PAEG) in Zusammenarbeit mit der AO Clinical Investigation and Documentation (AOCID) sowie der International Working-Group for Paediatric Traumatology (IAGKT) [3, 5],

- die Klassifikation der Mitglieder der Sektion der Kindertraumatologie der DGU und Lila (Licht und Lachen für Kranke Kinder; Effizienz in der Medizin e. V.) [1].

Im Folgenden wird die erstgenannte Klassifikation der AO-Gruppe vorgestellt. Sie basiert auf der ErwachsenenMüller-AO-Klassifikation [2] und berücksichtigt die zahlreichen kinderspezifischen Fraktureigenschaften. Für die Anwendung der AO-Paediatric benötigt man konventionelle Röntgenaufnahmen in lateraler und a.-p. Position. Der vollständige Frakturkode wird aus 5 bzw. 6 Kodes gebildet, in Abhängigkeit davon, ob ein Ausnahme- bzw. Zusatzkode angewendet wird (• Abb. 1).

\section{Einzelkodes der PAEG-Klassifikation}

\section{Knochenkode}

In Anlehnung an die AO-Klassifikation für Erwachsene werden die langen Röhrenknochen mit 1-4 bezeichnet ( 1 : Humerus, 2: Radius/Ulna, 3: Femur, 4: Tibia/ Fibula, - Abb. 2). Paarige Knochen mit identischem Verletzungsmuster werden durch einen Frakturkode klassifiziert. Ist z. B. nur ein Knochen am Unterarm bzw. Unterschenkel betroffen, wird dies durch einen entsprechenden Buchstaben ( $r, u$, $t, f)$ beschrieben. Betrifft die Verletzung beide Knochen mit unterschiedlichem Verletzungsmuster, muss jeder Knochen separat mit dem entsprechenden Buchstabenzusatz klassifiziert werden.

Trauma Berufskrankh 2010 - 12[Suppl 3]:323-328 DOI 10.1007/s10039-009-1586-y

C) Springer-Verlag 2010

\section{F.F. Fernandez $\cdot 0$. Eberhardt \\ Klassifikationen von Frakturen im Kindesalter}

\section{Zusammenfassung}

Eine Frakturklassifikation ist Grundlage der Erfassung epidemiologischer und klinischer Frakturdaten in einer systematischen einheitlichen Dokumentation und damit auch Grundvoraussetzung zur Evaluierung diagnostischer und therapeutischer Maßnahmen im Sinne einer Effizienz- und Effektivitätskontrolle. Mit der AO-Fraktur-Klassifikation des Erwachsenen konnten zwar auch bezüglich kindlicher Frakturen Erkenntnisse gewonnen werden, dennoch ist diese Einteilung nicht unverändert auf Brüche des wachsenden Skelettes übertragbar, da sowohl das Verletzungsmuster als auch Therapie und Outcome vom Alter und Reifestadium der

\section{Pediatric fracture classifications}

\section{Abstract}

A fracture classification forms the basis for compiling epidemiological and clinical fracture data in systematic and standardized documentation, thus forming the basic requirement for any evaluation of diagnostic and therapeutic measures in order to monitor efficiency and effectiveness. Information relating also to pediatric fractures can be gained using the $\mathrm{AO}$ fracture classification for adults, although this cannot be directly extrapolated to the growing skeleton, since injury patterns, therapy and outcome depend on growth plate age and stage of maturity.
Wachstumsfugen abhängen. Jüngst wurden 2 systematische kinderspezifische Klassifikationen erarbeitet und validiert, die Klassifikation der AO Paediatric Expert Group (PAEG) wird im vorliegenden Beitrag vorgestellt. Der vollständige Frakturkode wird aus 5 (Knochen-, Segment-, Typ-, Kinder- und Frakturschwerekode) bzw. 6 Kodes gebildet, in Abhängig-keit davon, ob ein Ausnahmebzw. Zusatzkode angewendet wird.

\section{Schlüsselwörter}

Kindliche Fraktur - Wachstumsfugen . Reifestadium · Frakturklassifikation . Frakturkode

Two child-specific classifications have recently been compiled and validated; one of these, the $\mathrm{AO}$ of the Paediatric Expert Group (PAEG) is presented here. The complete fracture code comprises between five (bone, segment, type, child and severity) or six codes, depending on whether an exception is made or an extra code is used.

\section{Keywords}

Pediatric fracture - Growth plates - Stage of maturity · Fracture classification · Fracture code 

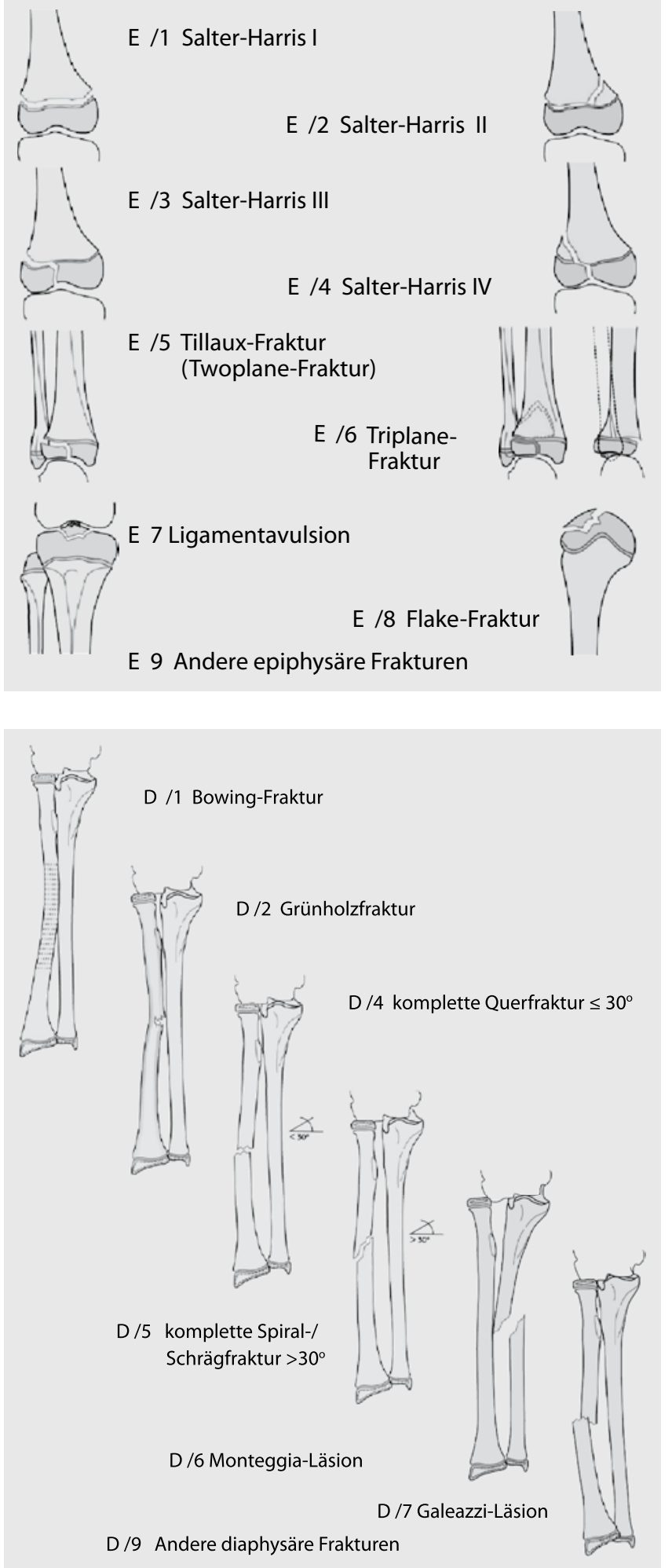

Ist das Verletzungsmuster bei paarigen Knochen identisch, wird die schwerwiegendere Fraktur angegeben.

\section{Segmentkode}

Die Röhrenknochen werden nach Segmenten eingeteilt in (• Abb. 2):

\section{Typkode}

Die Röhrenknochen werden auch nach Epi-, Dia- und Metaphyse differenziert (- Abb. 2), es wird also zwischen extra- und intraarkulären Frakturen unterschieden. Die epiphysären Frakturen stellen damit immer intraartikuläre Frakturen dar.

Die Größe der Metaphyse wird dadurch ermittelt, dass einen Quadrat über der gesamten Länge der Epiphysenfuge angelegt wird. Für die paarigen Knochen des Unterarms und Unterschenkels müssen beide Epiphysenfugen in dieses Quadrat eingeschlossen werden. Bei metaphysären Frakturen sollte die Mitte der Fraktur im Zentrum des Quadrats liegen
Abb. $4 \varangle$ Kindspezifische Einteilung der epiphysären Frakturen. (Aus [4])
Abb. $5<$ Klassifikation der diaphysären Frakturen. (Aus [4])
- Segment 1 (proximal): proximale Epiphyse und Metaphyse,

- Segment 2 (diaphysär): Diaphyse und

- Segment 3 (distal): distale Metaphyse und Epiphyse.

\section{Kinderkode}

In ihn fließen die speziellen Eigenschaften des wachsenden Skeletts ein, sie werden für die verschiedenen Frakturtypen in E, M und D eingeteilt.

Bei den metaphysären Frakturgeometrien werden 3 Typen unterschieden: Wulst-, Spiral- und Grünholzfrakturen mit $M / 2$. Die vollständigen Frakturen werden mit $\mathrm{M} / 3$ benannt ( $(\mathbf{A b b}$. 3) und die metaphysäre osteoligamentäre und einfache Avulsionsverletzung mit $\mathrm{M} / 7$ beziffert.

Die epiphysären Frakturen werden in den Kodes E/1-E/9 abgebildet, dabei entsprechen E/1-E/4 der Salter-HarrisKlassifikation (- Abb. 4). Weitere kinderspezifische Frakturen sind: TillauxFrakturen (E/5), Triplane-Fraktur (E7/6), Eminentia-intercondylaris-Avulsionsverletzung $(\mathrm{E} / 7)$ und Flake-Frakturen (E/8). Andere epiphysäre Frakturen werden in E/9 zusammengefasst.

Die Schaftfrakturen werden im Kinderkode unterschieden in

- Bowing-Frakturen (D/1),

- Grünholzfrakturen (D/2),

- Toddler-Frakturen (D/3),

- komplette Querfrakturen

(Winkel <30\%/D/4),

- komplette Spiral- und Schrägfrakturen (Winkel $>30 \% / 5$ ),

- Monteggia-Läsionen (D/6),

- Galeazzi-Läsionen (D/7). 


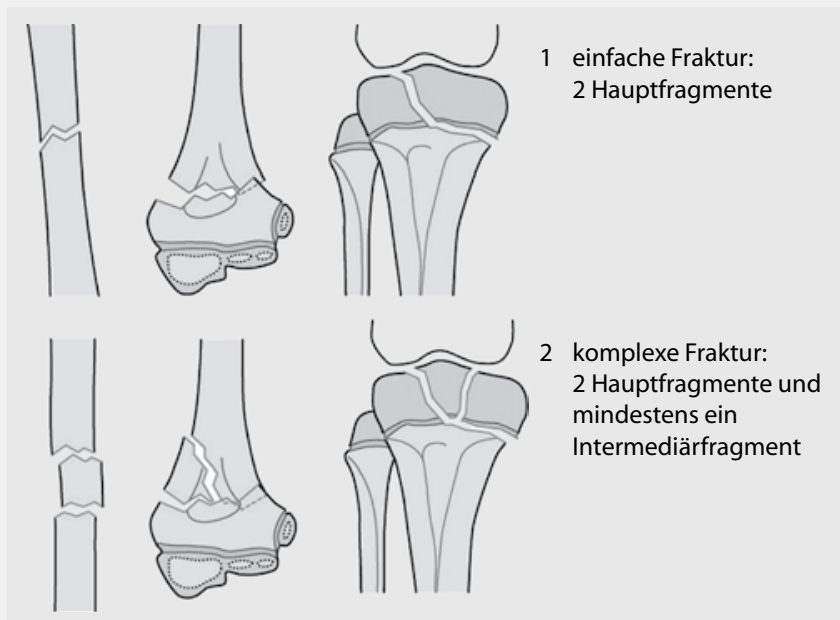

Abb. $6 \Delta$ Frakturschweregrad. (Aus [4])
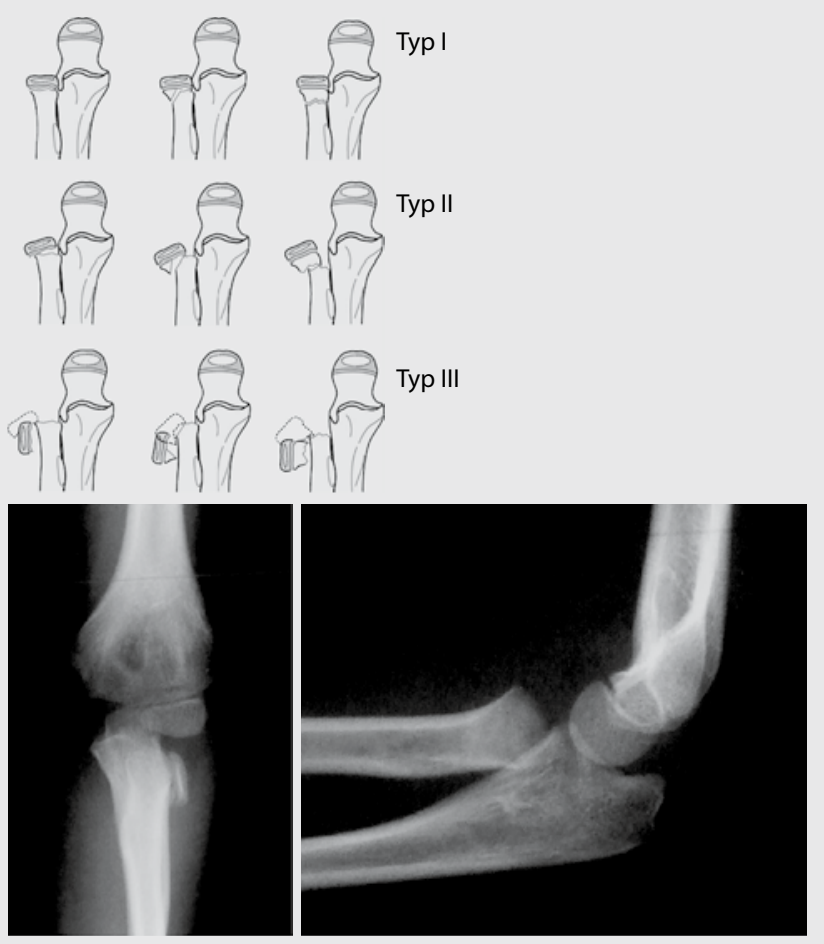

Abb. 8 Radiuskopf-/-halsfrakturen: Kodierung in Abhängigkeit von der Dislokation (Kode 21-E/1-2, 21-M/2-3 Typ I-III), Erläuterung s. Text. (Aus [4])
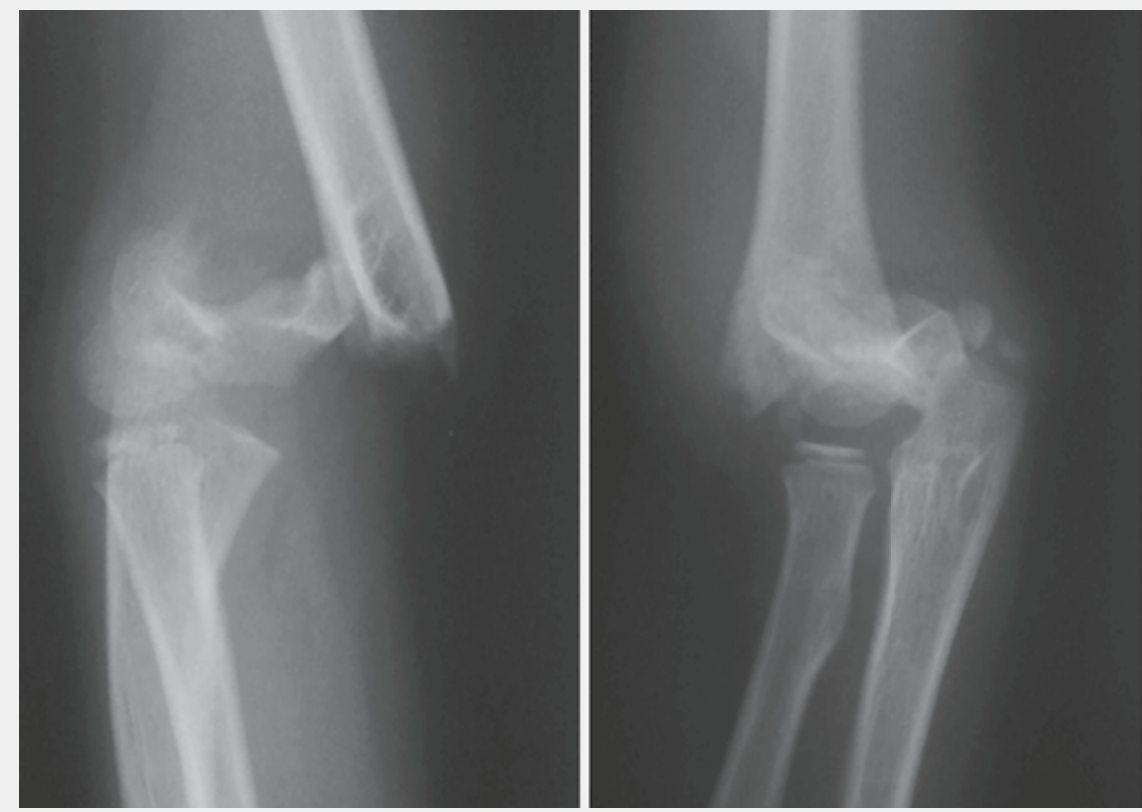

Abb. 7 Suprakondyläre Frakturen: Kodierung in Abhängigkeit von der Dislokation (Humerus, distal, Metaphyse, komplett, einfach, grob disloziert, Kode $13-\mathrm{M} / 3.1$, Typ I-IV)

D/9 bildet alle andere Schaftfrakturen ab, die keinem der genannten Frakturtypen zugeordnet werden können (• Abb. 5).

\section{Frakturschweregradkode}

Es werden 2 Grade unterschieden (- Abb. 6):

- Grad 1 für einfache Frakturen (partiell instabile Frakturen mit 3 Fragmenten einschließlich eines vollständig dislozierten Fragments) und
- Grad 2 für komplexe Frakturen (instabile Frakturen mit mehr als 3 Fragmenten).

\section{Ausnahme- und Zusatzkodes}

Mit der bisher vorgestellten Klassifikation können nicht alle Kinderfrakturen abgebildet werden. Daher wurden Zusatzund Ausnahmekodes entwickelt.
Übergangsfrakturen. Sie sind unabhängig vom metaphysären Keil zu den epiphysären Frakturen zu rechnen.

Knöcherne Bandverletzungen. Intraartikuläre knöcherne Bandverletzungen werden zu den epiphysären Frakturen und extraartikuläre zu den metaphysären Frakturen gezählt.

Apophysenfrakturen. Sie werden den metaphysären Frakturen zugeordnet. 


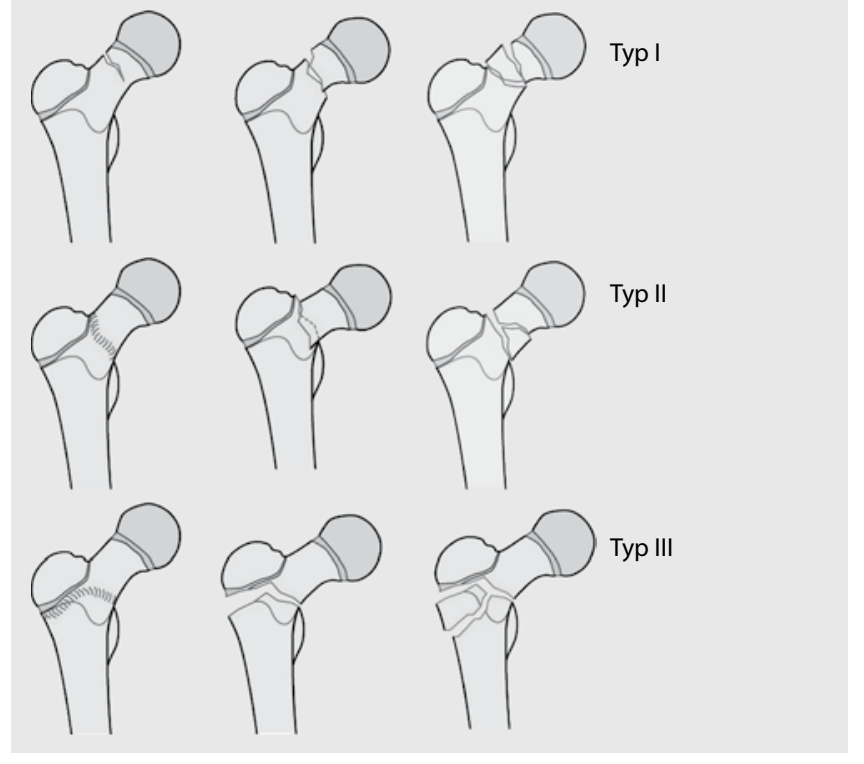

Abb. $9<$ Einteilung der Schenkelhalsfrakturen Typ I-III. (Aus [4])
Suprakondyläre Frakturen (13-M/3). Sie erhalten zusätzlich eine Ziffer (I-IV) für das Dislokationsausmaß nach von Laer et al. [1] ( $\bullet$ Abb. 7):

- Keine Dislokation: Grad I,

- Dislokation in einer Ebene: Grad II,

- Rotation mit Dislokation in 2 Ebenen: Grad III und

- Vollständige Dislokation ohne knöchernen Kontakt bzw. Fehlstellung in 3 Ebenen: Grad IV.

Radiushalsfrakturen (21-M/2 bzw. /3 oder 21-E/1 bzw. /2). Sie werden nach dem Ausmaß der Dislokation und der axialen Abweichung eingeteilt (• Abb. 8):

- Keine Dislokation oder Angulation: Typ I,

- Dislokation oder Angulation mit weniger als halber Schaftbreite: Typ II und

- Dislokation oder Angulation mit mehr als halber Schaftbreite: Typ II.I

Schenkelhalsfrakturen. Epiphysenlösungen ohne oder mit metaphysärem Keil werden nach der Salter-Harris-Klassifikation in E/1 bzw. E/2 differenziert (• Abb. 9).

Metaphysäre Schenkelhalsfrakturen werden unterschieden in:

- transzervikal: Grad I,

- basozervikal: Grad II und

- pertrochantär: Grad III.

\section{Fazit}

Mit der vorgestellten AO-PaediatricKlassifikation und der Klassifikation der Sektion der Kindertraumatologie der DGU und Lila stehen uns evaluierte kinderspezifische Klassifikationen zur Verfügung, die als Grundstein für die Dokumentation von Frakturen des wachsenden Skeletts dienen können. Die damit geschaffene einheitliche kinderspezifische Klassifikation ist Basis für Studien, mit deren Hilfe Aussagen über Therapieaufwand und Resultate unterschiedlicher Therapien oder Prognosen von Verletzungen getroffen werden können.

Inwieweit sich diese Klassifikationen im klinischen Alltag durchsetzen können bzw. ob sie alltagstauglich sind, bleibt abzuwarten. Für den klinischen Alltag benötigen wir prägnante, einfache Klassifikationen mit therapeutischer Relevanz.

\section{Korrespondenzadresse}

\section{Dr. F.F. Fernandez}

Orthopädische Klinik, Olgahospital, Klinikum Stuttgart,

Bismarckstraße 8, 70176 Stuttgart

f.fernandez@klinikum-stuttgart.de

Danksagung. Unseren Dank an Herrn Dr. Slongo und Herrn Audigé für Ihre Genehmigung, die Abbildungen aus ihrer Arbeit [4] benutzen zu dürfen.

Interessenkonflikt. Der korrespondierende Autor gibt an, dass kein Interessenkonflikt besteht.

\section{Literatur}

1. Laer L von, Gruber R, Dallek M et al (2000) Classification and documentation of children's fractures. Eur J Trauma 26:02-14

2. Müller ME, Nazarian S, Koch P, Schatzker J (1990) The comprehensive classification of fractures of long bones. Springer, Berlin Heidelberg New York

3. Slongo T, Audigé L (2007) Fracture and dislocation classification compendium for children. The AO Pediatric Comprehensive Classification of Long Bone Fractures (PCCF) on behalf of the $A O$ Pediatric Classification Group. J Orthop Trauma [Suppl] 21:S135-S160

4. Slongo T, Audigé L, AO Pediatric Classification Group (2007) AO pediatric comprehensive classification of long-bone fractures (PCCF). Brochure AO Education. AO Publishing, Dübendorf

5. Slongo T, Audige L, Clavert JM et al (2007) The AO comprehensive classification of pediatric longbone fractures. A web-based multicenter agreement study. J Pediatr Orthop 27:171-180 